\title{
Simultaneous HPTLC Estimation of Simvastatin and Ezetimibe in Tablet Dosage Form
}

\author{
BHUPENDRA SHRESTHA*, B. STEPHENRATHINARAJ ${ }^{\mathbb{I}}$, \\ SITA SHARAN PATEL ${ }^{\S}$, N.K. VERMA ${ }^{\#}$ and R. MAZUMDER \\ *Department of Pharmaceutical Chemistry, \\ Himalayan Pharmacy Institute, Majhitar, E.Sikkim-737136, India. \\ IIDepartment of Pharmaceutical Analysis, Vaagdevi College of Pharmacy, \\ Hanamkonda, Warangal, Andhra Pradesh, India. \\ ${ }^{\S}$ Department of Pharmacology, \\ Himalayan Pharmacy Institute, Majhitar, E.Sikkim-737136, India. \\ \#Department of Pharmacology, \\ Himalayan Pharmacy Institute, Majhitar, E.Sikkim-737136, India. \\ Department of Pharmaceutics, Calcutta Institute of Pharmaceutical Technology and \\ AHS, Howrah, West Bengal-711316, India. \\ shrestha2k@yahoo.com
}

Received 27 November 2009; Accepted 20 January 2010

\begin{abstract}
A method enabling the precise and quick simultaneous analysis of simvastatin and ezetimibe in tablet formulation by high performance thin layer chromatography has been presented. Samples of simvastatin and ezetimibe in organic solvents were separated on a plate coated with silica gel 60 F-254 and the chromatograms were developed using a mixture of chloroform and methanol (9.5: $0.5 \% \mathrm{v} / \mathrm{v})$. The method has a linearity range of $40-280 \mathrm{ng} \cdot \mathrm{mL}^{-1}$ for both the drugs when scanned at $254 \mathrm{~nm}$. The limit of detection and limit of quantitation was found to be 30 and 100 ng.band ${ }^{-1}$ respectively, for both the drugs. The mean percent recovery was found to be 100.65 and 101.55 for simvastatin and ezetimibe. The intra-day and inter-day precision studies were carried out with mean RSD of 0.88 and 1.27 for ezetimibe and 1.35 and 1.50 for simvastatin.
\end{abstract}

Keywords: Simultaneous, Simvastatin, Ezetimibe, HPTLC.

\section{Introduction}

Simvastatin (SIM) is chemically 2,2-dimethylbutanoic acid(1S,3R,7S,8S,8aR)-1,2,3,7,8,8ahexahydro-3,7-dimethyl-8-[2-[(2R,4R)-tetrahydro-6-oxo-2H-pyran-2-yl]ethyl]-1naphthalenyl 
ester $^{1}$. The chemical structure of SIM is shown in Figure 1. It is a fungal metabolite, lactone prodrug, that are modified in the liver to active hydroxy acid forms. SIM competitively inhibit conversion of HMG-CoA to mevalonate, a rate limiting step in cholesterol synthesis ${ }^{2}$. Ezetimibe (EZE), the first compound approved for lowering total and LDL-C levels that inhibits cholesterol absorption by enterocytes in the small intestine is used primarily as adjunctive therapy with statins. In clinical trials, Ezetimibe produced significant reduction in LDL cholesterol level both alone and when added to statin therapies ${ }^{3}$. Chemically it is 1-(4-fluorophenyl)-3(R)-[3(4fluorophenyl)-3(S)-hydroxypropyl]-4(S)-(4-hydroxyphenyl)-2-azetidinone.

Simvastatin and ezetimibe has been recently combined as a fixed dose therapy approved by US-FDA, which offers clinicians the opportunity to simultaneously inhibit two key pathways in cholesterol metabolism, hepatic cholesterol biosynthesis and the absorption of cholesterol at the level of the proximal jejunum.

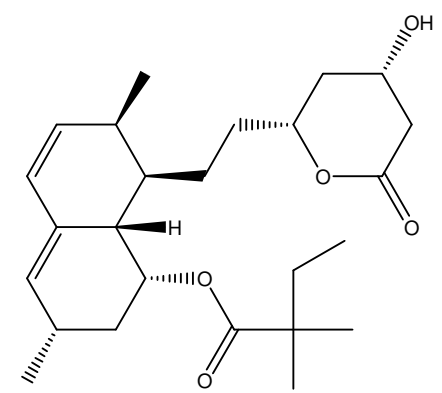

Figure 1. Chemical structure of Simvastatin.

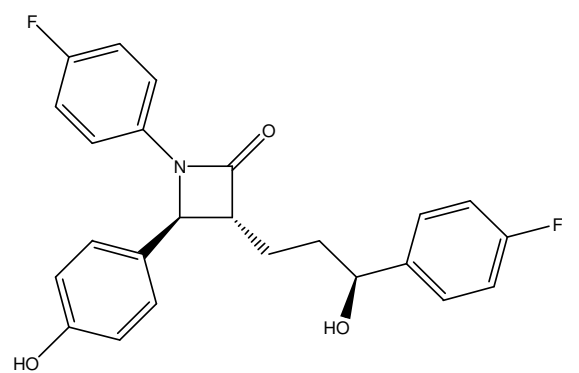

Figure 2. Chemical structure of Ezetimibe.

It was found that there is a method available for analysis of SIM by HPLC in combination with lovastatin ${ }^{4}$ and a HPTLC method ${ }^{5}$ as well for analysis of SIM. Sistla et.al $l^{6}$ has developed a HPLC method for analysis of EZE in pharmaceutical dosage forms. To our knowledge no article related to simultaneous high-performance thin-layer chromatography (HPTLC) determination of of SIM and EZE in tablet dosage forms has ever been published in literature. Now-a days HPTLC is becoming a routine analysis technique due to advantages of low operating cost, high sample throughput and need for minimum sample clean up. The major advantage of HPTLC is that several samples can be run simultaneously using a small quantity of mobile phase unlike HPLC, thus lowering analysis time and cost per analysis ${ }^{7,8}$.

The aim of the present work is to develop an accurate, precise, specific and repeatable HPTLC method for the simultaneous determination of SIM and EZE in tablet dosage form. The proposed method was validated as per ICH guidelines ${ }^{9,10}$ and its updated international convention ${ }^{11}$.

\section{Experimental}

Micro labs Pvt. Ltd. Hosur, Tamil Nadu, India kindly supplied pure drug sample of Simvastatin and Ezetimibe. All chemicals and reagents used were of analytical grade and were purchased from E.Merck (India) Limited, Mumbai. Marketed formulations used were Starstat-EZ tablets, Lupin Pharma and Simvas-EZ tablets, Micro Labs.

\section{Instrumentation}

The samples were spotted in the form of bands of width $6 \mathrm{~mm}$ with a Camag $100 \mu \mathrm{L}$ sample syringe (Hamilton, Bonaduz, Switzerland) on silica gel precoated aluminum plate $60 \mathrm{~F}-254,(20 \mathrm{~cm} \times 20 \mathrm{~cm})$ with $200 \mu \mathrm{m}$ thickness; E. Merck, Darmstadt, Germany, 
using a Camag LinomatV (Switzerland) sample applicator. The plates were pre-washed by methanol and activated at $110^{\circ} \mathrm{C}$ for $5 \mathrm{~min}$ prior to chromatography. The space between two bands was kept at $10 \mathrm{~mm}$. The slit dimension was kept at $4 \times 3 \mathrm{~mm}$ and $4.0 \mathrm{~mm} / \mathrm{s} \mathrm{scanning}$ speed was employed. The monochromator bandwidth was set at $10 \mathrm{~nm}$, each track was scanned thrice and baseline correction was used. The mobile phase consisted of Chloroform and Methanol in the ratio of 9.5: $0.5 \mathrm{v} / \mathrm{v}$. Linear ascending development was carried out in a twin trough glass chamber (Camag, Muttenz, Switzerland) saturated with the mobile phase. The optimized chamber saturation time for mobile phase was $30 \mathrm{~min}$ at room temperature $\left(25^{\circ} \mathrm{C} \pm 2\right)$ at relative humidity of $55 \% \pm 5$. Subsequent to the development; TLC plates were dried in current of air with the help of air dryer. Densitometric scanning was performed on Camag TLC scanner II in the absorbance-reflectance mode at $254 \mathrm{~nm}$ using CATS software. The source of radiation utilized was deuterium lamp emitting continuous UV spectrum between 190 and $400 \mathrm{~nm}$. Concentrations of the compound chromatographed were determined using densitogram area.

\section{Standard solution preparation}

About $100 \mathrm{mg}$ of SIM and EZE were transferred to $100 \mathrm{~mL}$ volumetric flasks separately, dissolved with methanol and made up to $100 \mathrm{~mL}$ with the same solvent. From this $10 \mathrm{~mL}$ was pippetted out into a $50 \mathrm{~mL}$ volumetric flask and made up the volume with methanol to give a concentration of $200 \mu \mathrm{g} \cdot \mathrm{mL}^{-1}\left(1000 \mathrm{ng} \cdot \mu \mathrm{L}^{-1}\right)$.

\section{Sample solution preparation}

Two different formulations were chosen for the analysis (Starstat-EZ tablets and Simvas-EZ tablets). Twenty tablets, each labeled to contain SIM $10 \mathrm{mg}$ and EZE $10 \mathrm{mg}$ per tablet, were weighed accurately, crushed and ground to a fine powder. An accurately weighed quantity of tablet powder equivalent to $100 \mathrm{mg}$ of SIM and EZE were transferred to a $100 \mathrm{~mL}$ volumetric flask containing $50 \mathrm{~mL}$ of methanol, sonicated for about $15 \mathrm{~min}$ and the volume was made up with methanol. The solution was then filtered through whatman filter paper No.41.First $5 \mathrm{~mL}$ of solution is discarded. Then $10 \mathrm{~mL}$ of the solution is pipetted out into a $50 \mathrm{~mL}$ volumetric flask and made up to $50 \mathrm{~mL}$ with methanol. From the resulting solution $5 \mu \mathrm{L}$ was spotted as sharp bands on the chromatoplate using Linomat IV spotting system. The chromatoplate was then developed in a pre-saturated twin trough chamber containing mobile phase. After development, the bands of the drugs were scanned at $254 \mathrm{~nm}$ using a densitometer. The peak area of standard and samples were used to calculate the amount of SIM and EZE present per tablets.

Validation of the method

\section{Linearity and range}

The standard solutions of SIM and EZE were prepared to reach a concentration range of 40-280 ng. $\mu \mathrm{L}^{-1}$ each. Five micro liters from each standard solution was spotted on the TLC plate to obtain final concentration of 200-1400 ng.band ${ }^{-1}$. Each concentration was spotted three times on the TLC plate. The peak areas were plotted against the corresponding concentrations to obtain the calibration graphs.

\section{Precision}

Precision of the method was verified by repeatability and intermediate precision studies. Repeatability studies were performed by analysis of three different concentrations (400, 600, 1000 ng.band $^{-1}$ ) of the drug, each concentration injected six times on the same day. Intermediate precision of the method was checked by repeating studies on three different days. 


\section{Limit of detection and limit of quantitaiton}

The limit of detection (LOD) and limit of quantitation (LOQ) was determined on the basis of signal to noise ratio. LOD was the amount of the applied sample producing a peak area that is equal to the sum of the mean blank area and three times the standard deviation. LOQ was the amount of the applied sample producing a peak area that is equal to the sum of the mean blank area and ten times its standard deviation.

\section{Robustness of the method}

Small changes in the mobile phase composition $( \pm 0.1 \mathrm{~mL}$ for each component) were made and the effects on the results were examined. Time from spotting to chromatography and from chromatography to scanning was varied by $\pm 15 \mathrm{~min}$.

\section{Accuracy}

Accuracy was determined by recovery studies. It was carried out by spiking $80 \%, 100 \%$ and $120 \%$ of the standard drugs to the pre-analysed marketed sample of SIM and EZE. Three determinations were performed at each level.

\section{Stability in sample solution}

Solutions at analytical concentrations were prepared from the sample solution and stored at room temperature for $0.5,1,3,6,9,12$ and $24 \mathrm{~h}$ in tightly capped volumetric flasks, protected from light, on a laboratory bench. They were then applied to a TLC plate and analyzed by the developed method for the presence of any band other than that of the drug.

\section{Results and Discussion}

Finding the optimum mobile phase

The TLC procedure was optimized with the objective of developing a precise, robust and accurate assay method. Both the pure drugs and the samples were applied to TLC plates and chromatographed with different mobile phases. Initially, chloroform-ethyl acetate 5:5(v/v) and $n$-hexane chloroform $4: 6(\mathrm{v} / \mathrm{v})$ were used. The best results were obtained by use of chloroform-methanol in the ratio of 9.5: $0.5(\mathrm{v} / \mathrm{v})$. RF values were 0.30 for EZE and 0.42 for SIM (Figure). This mobile phase enabled good resolution of SIM and EZE from the sample solution.

\section{Method Validation}

\section{Linearity and range}

Linearity was observed over the concentration range of 200-1,400 ng.band ${ }^{-1}$. The linearity of the calibration plots was confirmed by the high value of the correlation coefficients $\left(r^{2}=0.9989\right.$ for SIM and 0.9988 for EZE).

Table 1. Linear regression data

\begin{tabular}{ccccc}
\hline Drug & Linearity range, ng.band & $\mathrm{r}^{2}$ & Slope & Intercept \\
\hline SIM & $200-1400$ & 0.9989 & 5.591 & 20.301 \\
EZE & $200-1400$ & 0.9988 & 5.776 & 13.173 \\
\hline
\end{tabular}

\section{Precision}

The repeatability of measurement of peak area was expressed as RSD. The intra-day and interday variation of SIM and EZE at three different concentrations - 400, 600 and 1,000 ng. band ${ }^{-1}$ was given in Table 2 . The RSD for intra and inter day analysis was always less than $2 \%$. These low values of the RSD show the repeatability and precision of the method were very good. 
Table 2. Intra-day and inter-day precision of the method

\begin{tabular}{ccccccc}
\hline Amount & \multicolumn{9}{c}{ SIM } \\
\cline { 2 - 7 } ng.band $^{-1}$ & Mean & SD & RSD\% & Mean & SD & RSD\% \\
\hline \multicolumn{7}{c}{ Intra-day precision } \\
400 & 2372.116 & 29.36 & 1.24 & 2634.828 & 35.35 & 1.34 \\
600 & 3457.413 & 55.33 & 1.60 & 3923.930 & 34.69 & 0.88 \\
1000 & 5667.524 & 69.46 & 1.23 & 6491.904 & 28.07 & 0.43 \\
\multicolumn{7}{c}{ Inter-day precision } \\
400 & 2382.046 & 32.78 & 1.38 & 2667.266 & 47.34 & 1.77 \\
600 & 3437.340 & 61.68 & 1.79 & 4037.211 & 56.99 & 1.41 \\
1000 & 5652.860 & 75.55 & 1.34 & 6481.567 & 42.31 & 0.65 \\
\hline
\end{tabular}

\section{Robustness of the method}

The robustness of the method was established by the low values of RSD of peak areas for each set of conditions. It is presented in Table 3.

Table 3. Results from robustness testing of the method for SIM and EZE

\begin{tabular}{lcc}
\hline \multirow{2}{*}{ Condition } & \multicolumn{2}{c}{ RSD\% } \\
\cline { 2 - 3 } & SIM & EZE \\
\hline Mobile phase composition & 1.89 & 1.72 \\
Time from sample application to chromatography & 2.01 & 1.84 \\
Time from chromatography to scanning & 1.76 & 1.24 \\
\hline
\end{tabular}

\section{Limit of detection and limit of quantitation}

The LOD and LOQ for a signal to noise ratio of 3:1 and 10:1 was found to be 30 and 80 ng.band $^{-1}$ for SIM and EZE which indicates the method has sufficient sensitivity.

\section{Accuracy}

The recovery of SIM and EZE from the formulation was determined by comparing the peak areas obtained from pre-analyzed formulation. The mean percent recovery was found to be $100.65 \& 101.55$ for SIM and EZE. The results are shown in Table 4.

Table 4. Results from recovery studies

\begin{tabular}{cccc}
\hline Drug & Amount of drug added, mg & Amount obtained, mg & Recovery, \% \\
\hline \multirow{2}{*}{ SIM, 10 mg } & 8 & 17.69 & 98.27 \\
& 10 & 20.40 & 102 \\
& 12 & 22.37 & 101.68 \\
EZE, $10 \mathrm{mg}$ & 8 & 18.16 & 100.88 \\
& 10 & 20.34 & 101.7 \\
& 12 & 22.46 & 102.09 \\
\hline
\end{tabular}

Stability in sample solution

There was decrease in drug concentration over time which means that the drug is not stable in solution. Therefore it is suggested that fresh solutions of SIM and EZE to be prepared for both the standard and sample. 


\section{References}

1. Moffat A C, Osselton D M and Widdop B, Eds., Clarke's Analysis of Drugs and Poisons, $3^{\text {rd }}$ Ed., Pharmaceutical Press, London (UK), 2004.

2. Brunton, L L, Lazo J S and Parker K L, Eds., Goodman and Gilman's, The Pharmacological Basis of Therapeutics, $11^{\text {th }}$ Ed., The McGraw Hill Co., 2006.

3. Simard C and Turgeon J, Can J Clin Pharm., 2003, 10, 13A-20A.

4. $\quad$ Srinivasu M K, Narasa R A and Rao D S, Indian Drugs, 2004, 41(3),156-159.

5. Suresh B, Meyyanathan S N and Ramasarma G V S, Ars Pharm., 2004, 45(2), 121-129.

6. Sistla R, Tata V S S K, Kashyap Y V, Chandrasekar D and Diwan P V, J Pharm Biomed Anal., 2005, 39(3), 517-522.

7. Sethi P D, High Performance Thin Layer Chromatography, Quantitative Analysis of Pharmaceutical Formulations, $2^{\text {nd }}$ Ed., CBS Publishers and distributors, New Delhi, India, 1996.

8. Thin Layer Chromatography, Monograph 2.2.27, European Pharmacopoeia, Council of Europe, 2002.

9. ICH, Q2A Validation of Analytical Procedure: Methodology International Conference on Harmonization, Geneva, October 1994.

10. ICH, Q2B Validation of Analytical Procedure: Methodology International Conference on Harmonization, Geneva, March 1996.

11. ICH Guidance on Analytical Method Validation, in: Proceedings of the International Convention on Quality for the Pharmaceutical Industry, Toronto, Canada, September, 2002. 


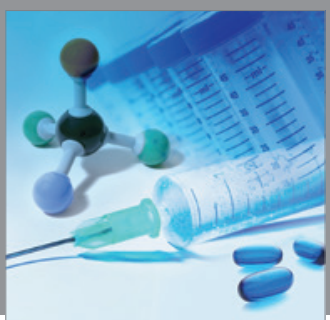

International Journal of

Medicinal Chemistry

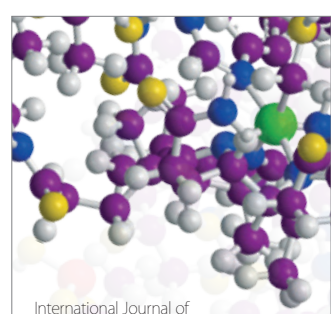

Carbohydrate Chemistry

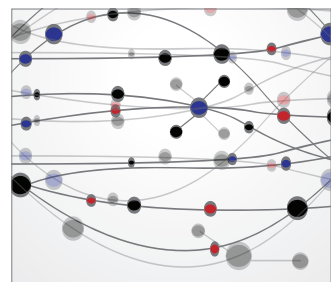

The Scientific World Journal
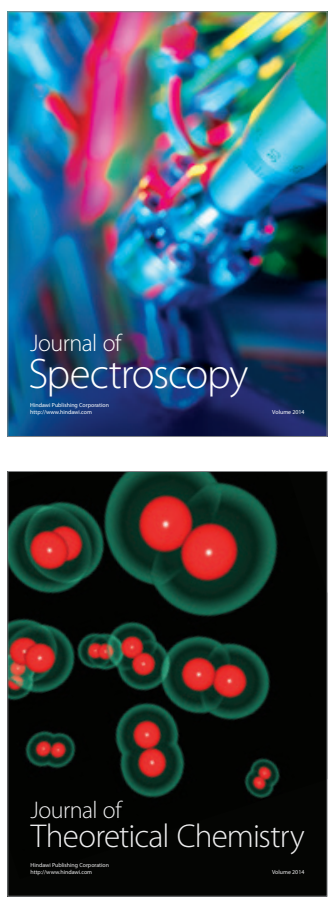
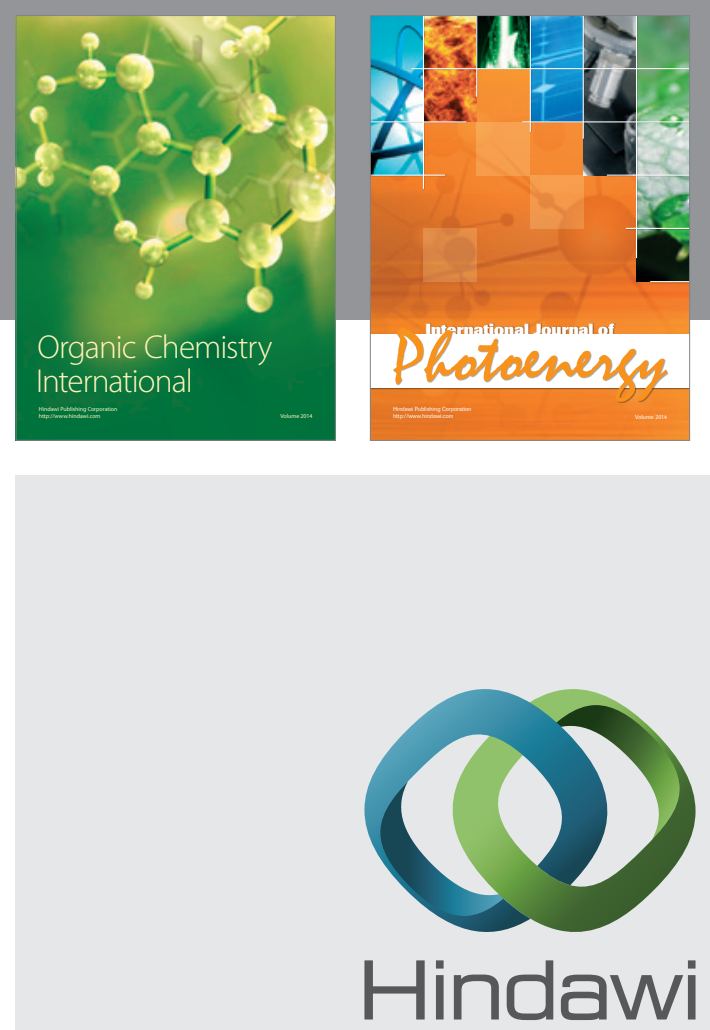

Submit your manuscripts at

http://www.hindawi.com
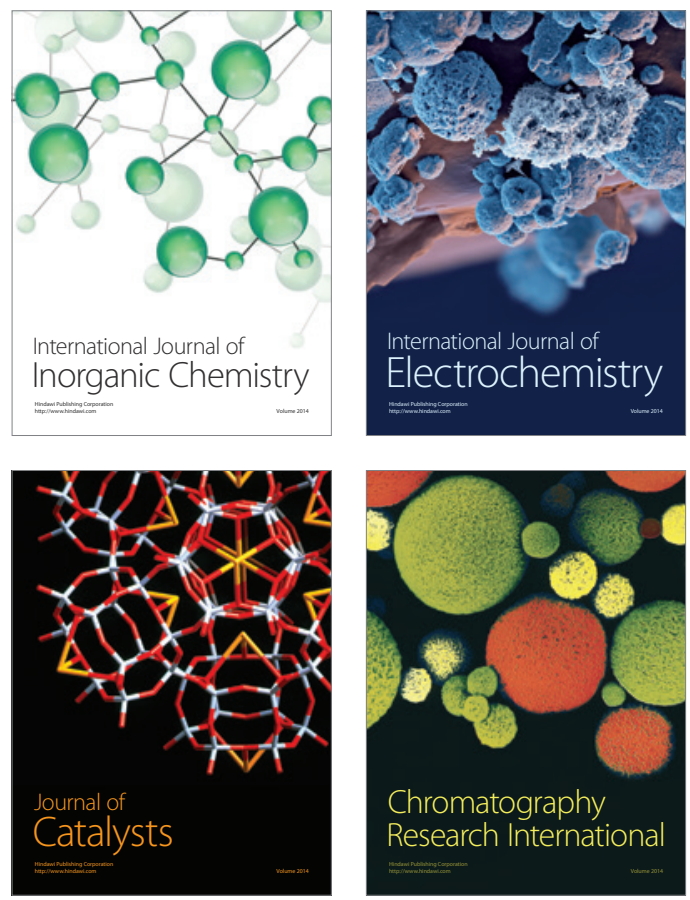
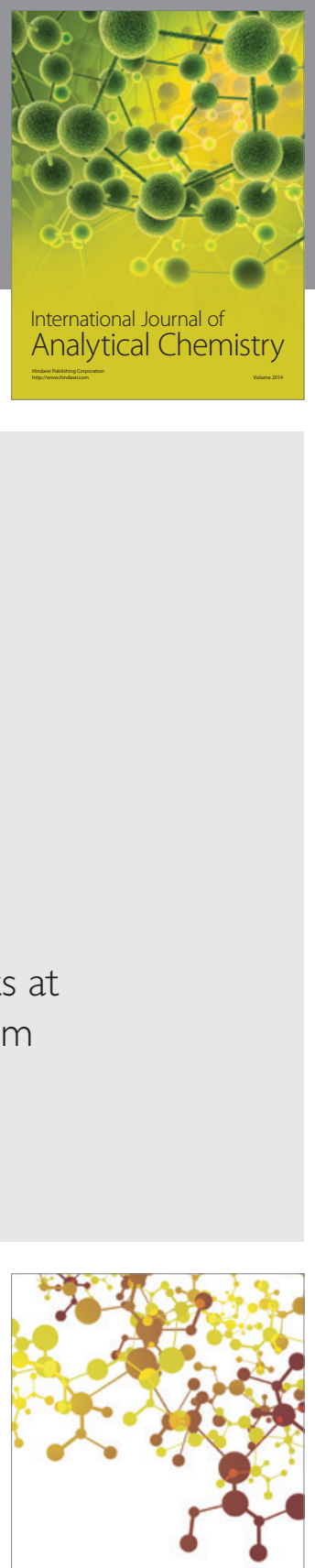

Journal of

Applied Chemistry
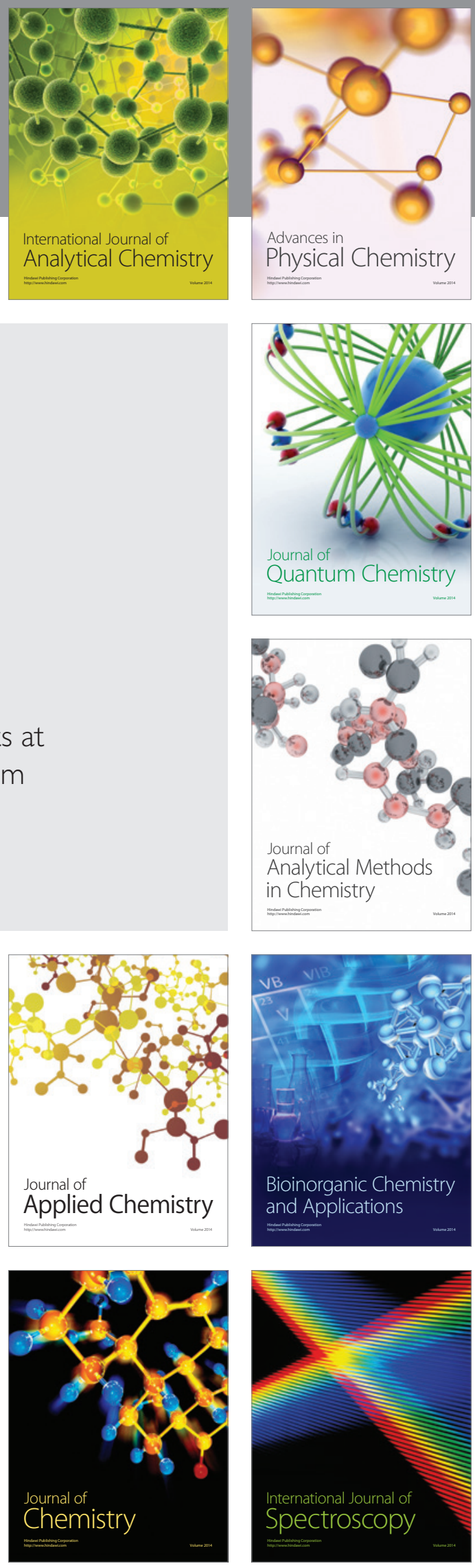